Review Article

\title{
The Historical Development of Deqi Concept from Classics of Traditional Chinese Medicine to Modern Research: Exploitation of the Connotation of Deqi in Chinese Medicine
}

\author{
Hong-Wen Yuan, ${ }^{1,2}$ Liang-Xiao Ma, ${ }^{1,3}$ Dan-Dan Qi, ${ }^{1}$ Peng Zhang, \\ Chun-Hua $\mathrm{Li}^{4}{ }^{4}$ and Jiang $\mathrm{Zhu}{ }^{1,3}$ \\ ${ }^{1}$ School of Acupuncture Moxibustion and Tuina, Beijing University of Chinese Medicine, Beijing 100029, China \\ ${ }^{2}$ School of Traditional Chinese Medicine, Capital Medical University, Beijing 100069, China \\ ${ }^{3}$ The Key Unit of Evaluation of Characteristic Acupuncture Therapy, State Administration of Traditional Chinese Medicine, \\ Beijing 100029, China \\ ${ }^{4}$ Beijing Electric Power Hospital, Capital Medical University, Beijing 100073, China
}

Correspondence should be addressed to Jiang Zhu; jzhjzh@263.net

Received 12 May 2013; Revised 11 September 2013; Accepted 18 September 2013

Academic Editor: Lijun Bai

Copyright ( 2013 Hong-Wen Yuan et al. This is an open access article distributed under the Creative Commons Attribution License, which permits unrestricted use, distribution, and reproduction in any medium, provided the original work is properly cited.

\begin{abstract}
Although it is difficult in fully clarifying its mechanisms and effects, Deqi still can be considered as an instant "sign" of acupuncture response of the patient and acupuncturist, which has a significant value in clinic and research. This paper aims to take a history trace to the development of Deqi theory, understand the connotation of Deqi based on Chinese medicine theory, and establish an evaluation methodology accordingly. We believe that Deqi is not only the needling sensation, but also the perception of changes of $q i$ ' flowing of the patient elicited by needling on acupoints. The signs of Deqi include the patient's subjective perception (needling sensation), the objective physiological changes (common referred to the skin redness around the acupoints and the response of brain), and the acupuncturists' perception. Although Deqi is essential for attaining the effect, it may not be the necessary sign of the ideal efficacy. It is found that the characteristics of Deqi sensations, Deqi's intensity, time duration, and the propagation will all affect the efficacy. Thus, acupuncturists should pay attention to elicit and control Deqi state, which is also the key point in modern research on the therapeutic implications of Deqi.
\end{abstract}

\section{Introduction}

There is so far little evidence of investigating the relationship between the therapeutic efficacy of acupuncture and the Deqi experience. However, as the specific perception of acupuncture stimulation, Deqi may influence the physical and psychological condition of the patients.

The terms of "Deqi" and "Qizhi" were first found in "Huang Di Neijing" [1]. The sentence "acupuncture therapy does not take effect until the arrival of energy," which is thought to be the basic principle to guide the clinical practice of acupuncture. At present, most acupuncture doctors and researchers in China [2-5] believe that Deqi is essential for the efficacy of acupuncture and it is necessary to induce Deqi sensation to a certain degree.
Some clinical results confirm that Deqi reveals some connection with the efficacy of acupuncture treatment [6-11]; however, others concluded that Deqi sensations did not result in effectiveness $[12,13]$. Learning from these studies, we found that studies conducted in China usually applied deep needling ( 1 to $2 \mathrm{~cm}$ ) with manipulations every $5 \mathrm{~min}$ during needle retention ( $30 \mathrm{~min}$ as usual), so as to maintain certain intensity and time duration of Deqi sensations. While the studies abroad mostly insert superficially or manipulate needle only once in the treatment.

There is one sentence in Neijing that talked about Deqi: "The $q i$ in acupoints is delicate, means that to elicit responses of $q i$ (Deqi), maintaining $q i$, and watch the movements of energy is an important step in acupuncture therapy" 
(from Ling Shu Chapter 3 on minute needle). It confirmed that "maintaining qi" actually refers to the fact that maintaining the intensity and time duration of needling sensation is necessary after Deqi. The manipulation every 5 min during needle retention after Deqi may be the key to a higher efficacy in the study. Some researchers also pay attention to the dose-effect relationship of acupuncture and consider that the efficacy of acupuncture treatment depends on complex factors, such as the number of needles, depth of needling, point location, needling retention, and interval between two sessions [14]. Therefore, the intensity of needling in most present acupuncture studies might not be enough for achieving therapeutic effects. It may help some confounders to have biased findings toward a negative outcome $[15,16]$. These views support that Deqi is a key to the treatment efficacy. However, the diversity of perspectives on the relationship between Deqi and effect in current studies may be caused by different understanding of Deqi.

The distinct difference in the understanding of Deqi can be found in the literatures in China and other countries. Among the Deqi related literatures published in China since 1950, there are 137 articles (67\%) discussed on the understanding of Deqi. There are a variety of definitions of Deqi across different textbooks.

\section{The Definitions of Deqi Nowadays}

In the latest "Eleventh Five-Year" national plan textbook "Acupuncture and Moxibustion," Zhen Jiu Xue [17] states: "Deqi, normally called Qizhi in ancient, or needling sensation in modern, refers to the response of channel qi elicited by acupuncture stimulation such as lifting, thrusting, as well as rotating the needle after inserting the needle into an acupoint." The signs of Deqi include two aspects, one is the patients' needling sensations, and the other is acupuncturist's perceptions. When obtaining $q i$, the patient may feel sensations of suan (soreness), ma (numbness), zhang (fullness/distention), and zhong (heaviness) and sometimes a feeling of heat, cold, pain, itching, muscular twitching, formication, and so forth, and those sensations can spread in some certain directions. A few patients may show the reaction of twitching or involuntary movement of skin and muscles along the stimulated channel, or red skin rash, or red or white lines on the skin around the punctured area. At the same time, the acupuncturist may feel heavy, tight, or vibration of the needle. If Deqi is not evoked, the patient would not have any special feeling or reactions, and the acupuncturist may feel the needles are loose and empty.

The widely accepted definition of Deqi in English language textbooks is: "Traditional acupuncture involves stimulation with very fine needles inserted into defined sites on the body, eliciting a composite of sensations, termed Deqi, which is considered to be related to clinical efficacy in traditional Chinese medicine" [18].

The latest Chinese-English Dictionary of Traditional Chinese Medicine (TCM) defines Deqi as "needling sensation, which refers to the patient's response to sore, numb, distention, electric shock and the doctor's heavy and tight sensation coming from beneath the needle" [19].
Several randomized controlled clinical trials in a large sample with a significant influence usually defined Deqi as "an irradiating feeling deemed to indicate effective needling" $[6,20,21]$.

Recently, the most common definition of Deqi is "the needling sensations of both patient and acupuncturist." However, in the theory of TCM acupuncture, needling sensation is the most significant manifestation of Deqi, but they are not equal on the level of connotation. In addition, modern functional brain imaging technology also demonstrated that classic Deqi sensation, such as a sense of soreness, had different effect on the certain area of the brain compared with sharp pain [22] which further supports the point that Deqi is not equal to needling sensation.

\section{The Origin of Deqi Theory}

The theory of TCM acupuncture generated from traditional Chinese culture. Therefore, prior to the study of Deqi theory, we should firstly understand its background, and then the connotation of Deqi could be explained by modern research methods.

Deqi is derived from the concept of "Q $i$ " in "Huang $D i$ Neijing" (written in AD 206 221 [23]). "Qi" is an important concept in Neijing to describe the activities of human life. The ability to maintain a healthy state is normally called healthy $q i$ (zheng qi) or grain qi ( $g u q i$ ), while the exogenous pathogenic factors that lead to varieties of diseases or pathological changes in the body are called pathogenic qi (xie qi).

In Neijing, the formation of the theory of " $Q i$ " is mainly originated from "the theory of qi transformation in life" in Taoism. In the Taoism work "Huai Nan Zi" [24], "body", "qi", and "mind" are composed of three treasures of life. They are interdependent and mutually related. The efficacy of acupuncture would be closely related to the patient's mental status and psychological factors, showing correspondence between " $q i$ " and "mind". Both the patient and acupuncturist will perceive certain sensations after Deqi, which is actually the interactions between "qi" and "body". Moreover, TCM holds that the $q i$ of the human body corresponds to the seasons, resulting in the relationship between physiological changes and the replacement of the four seasons. Thus, the seasons may affect the difficulty of Deqi. For example, the blood flows superficially in summer, during which Deqi is easier, while the blood flows deeply in winter and Deqi is relatively difficult. In Neijing, "qi" can also be classified into three categories: organ's qi (Zangqi), channel's qi (Jingqi), and vessel's $q i$ (Maiqi). These three types of $q i$ circulate in the body nonstoppingly. Therefore, stimulating acupoints on channel can regulate the certain internal organ to cure the disease. Detecting the changes of pulse can also be applied to determine "Deqi" in Neijing.

In summary, the theory of "Q $i$ " in Neijing is the basis of the traditional acupuncture theory of "Deqi". Phenomenon and impact of Deqi are all developed on this basis. TCM acupuncture emphasizes that the acupuncturist should concentrate on the needling process and carefully perceive the sensations below the needle to judge and regulate $q i$ of the patient. 


\section{The Development of Connotation of Deqi}

The view that acupuncture treatment efficacy is closely related to Deqi originated in Neijing. "If insertion of needle fails to bring about the response of energy, treatment should be continued for as long as necessary. Acupuncture therapy does not take effect until arrival of energy (Qizhi)." (from Lingshu Chapter 1 Nine needles and twelve original points). What is more, there is a detailed explanation of "Qizhi" in Neijing, "the principles of needling dictate that needling should stop as soon as energy is brought into harmony, due to tone up the body energy of $y$ in and sedate the pathogen of $y a n g$, or to tone up the body energy of yang and sedate the pathogen of yin. ... The assertion that needling takes effect so long as Qizhi means that an excessive disease could be sedated and the deficient energy may be complement." (from Lingshu Chapter 9 From beginning to end). It expresses that the function of acupuncture is "regulating qi," which can be achieved by supplementing and draining acupuncture manipulation. Therefore, pathogens can be eliminated, deficient healthy $q i$ can be supplemented, or reversed flow of $q i$ be adjusted, leading to a state of yin-yang balance. The connotation of " $q i$ " in "Qizhi" refers to healthy $q i$ or grain qi. Obviously, Qizhi indicates "proper intensity of stimulation" and also the sign of removing the needle. In Neijing, "Deqi" and "Qizhi" are usually considered as the same, regarded as a sign of efficacy $[25,26]$.

The Difficult Classic (Nanjing) [27] written in AD 106 210 [28] normally has similar reputation as Neijing in Chinese medicine. The chapter of Seventy-eight Difficult Issues states, "Insert a needle along the route of the channel to induce Deqi firstly. Then thrusting the needle inward is a supplementary method, while lifting the needle outward is a draining method." That text shows that certain needling techniques for supplementary or draining are applied after Deqi. Nanjing describes Deqi as the basis and premise of acupuncture manipulation. It may indicate that Deqi and Qizhi are not the same. Deqi refers to the first period of Qizhi, which is the sign of ideal efficacy.

The development of acupuncture can be traced to the Jin and Yuan Dynasty (AD 1115 1368), clarifying that Deqi is the base of acupuncture manipulation. There is one sentence in Song to Elucidate Mysteries (Biao You Fu) written by Dou Hanqing starting, "Then the acupuncturist should perceive $q i$ carefully. The sensation of loose and empty beneath the needle means $q i$ does not arrive. While heaviness, tightness and fullness sensations suggest that $q i$ has arrived. When $q i$ arrives, manipulate the needle properly according to cold or heat syndromes; when $q i$ does not arrive, wait for $q i$ according to deficiency or excess conditions." It keeps the same point as the Nanjing. Till that time, it is found that Deqi not only is related to the treatment efficacy, but also can be used to determine the prognosis of the disease. As written in Song to elucidate mysteries, "the more quickly of $q i$ arrives, the easier the disease is to cure; supposing the qi does not arrive, the patient may be hard to cure," which means that the degree of difficulties of getting Deqi can predict the efficacy. Of course, here, the efficacy does not refer to the real-time acupuncture effect but to the significant treatment efficacy of the disease.
As the acupuncture developed in the Ming and Qing dynasties (1368 1911), there is a further illustration of the relationship between the acupuncturist's perception of the needle and efficacy. One of the most influenced publications at that time, The Complete Compendium of Acupuncture and Moxibustion (Zhen Jiu Da Cheng) [29], states, "How to remove the needle depends on the acupuncturist's perception on the needle. Extremely tense and firm sensation beneath the needle indicates the needle is being grabbed by pathogens, not by the healthy $q i$, therefore, the needle cannot be removed. If the needle was removed at this moment, the disease might be palindromia. Instead, needling techniques for supplementary and draining should be applied and retain the needle for a certain period. Only when a looser sensation beneath the needle is felt, the needle can be removed." According to these texts, acupuncturist should clarify the sensations beneath the needle after Deqi. It is called "distinguishing qi."

Originating from Nanjing, the connotation of "Deqi" and "Qizhi" are different; "Qizhi" is obtaining the effect of the final state through the returning of $q i$ in human body by normal acupuncture treatment, while "Deqi" is the early part of "Qizhi," which means that the qi has been elicited to the needle. After realizing Deqi, it is still needed to distinguish the character of $q i$ and apply the related manipulation or retain the needle, so as to reach the "Qizhi" state [30-33].

\section{The Evaluation Method of Deqi Based on Its TCM Connotation}

5.1. Both Notice the Perception of Patient and Acupuncturist. Evaluation of Deqi in ancient Chinese medicine mainly focused on the acupuncturist's perceptions, rather than the patient's needling sensation during an acupuncture treatment. Ancient Chinese acupuncturists believe that during acupuncture, the acupuncturist should concentrate on the changes of $q i$ after the needle being inserted, so as to know the situation of patient and disease, then give appropriate needling manipulations to regulate $q i$. Moreover, the ability of "eliciting qi," "distinguishing $q i$, ," and "regulating qi" reflects the level of healing skill of the acupuncturist. In modern time, Deqi is defined as the needling sensation, so the quantitative evaluations of Deqi are mainly based on the patient's needling sensations [34-37]. Although the needling sensations of the patient are more direct and sensitive, we suggest that the perceptions of the acupuncturist should be noticed as well, which is the key of "regulating qi with the needle."

5.2. Distinguish the Sensation of Penetrating Skin from Deqi. In TCM, Deqi is the sign of the change of $q i$, so it should be elicited when the needle is inserted into the acupoint. Park et al. [38] also find a strong connection between acupuncture sensation and tissue depth; the frequency of prinking and sharp sensation was significantly greater in shallower tissue levels, and the frequency of sensations described as traditional Deqi sensation, such as dull, heavy, and spreading, was significantly greater in deeper tissue levels. Therefore, the pain or other sensations which could be felt when 
the needle penetrated skin is not Deqi sensation. In recent needle sensation questionnaire/scale, the item "sharp pain," "pricking," and "penetrating" may evaluate the sensation of penetrating skin but not Deqi. It would be better to design an instrument for calling attention to the patient to distinguish the sensation of needling penetrating from Deqi.

\subsection{Evaluating Deqi More Comprehensively Than Existence} and Intensity. Deqi is closely related to the treatment efficacy in Neijing; however, the formation of Deqi is only the first step of achieving the ideal efficacy in traditional theory. Through diagnosing based on $q i$ after Deqi, retaining the needle skills further improve the acupuncture efficacy. After immediately finishing the treatment after Deqi, the patient condition would deteriorate as the doss of stimulation is not enough. It was also stated that " $Q i$ extending affected treatment partially" as mentioned in Zhen Jiu Da Cheng, which means that the correct direction and a certain distance of Deqi spreading can enhance the efficacy. Therefore, the evaluation of Deqi existence and intensity is only two aspects in evaluating the relationship between the Deqi and the treatment efficacy. The nature of qi, intensity, duration, and the spreading should be recorded comprehensively by Deqi evaluation instrument, which may influence the efficacy in clinical trials.

5.4. Notice the Influence of Deqi in "Mind". The traditional theory of Deqi does not merely refer to the needle feeling, but also includes the reaction of " $q i$ " in the human body. According to TCM theory, this phenomenon can affect constituting the other two significant elements of the human body: the "body" and "mind;" manifested in "body" is the needle feeling, while that manifested in "mind" referred to the change of the state of brain function, which are the two most important factors in evaluating the Deqi.

The study of brain central mechanisms of acupuncture carried out recently is combined with a large number of brain imaging technology (PET, SPECT, fMRI, etc.), which provides a scientific method in investigating the Deqi effect. Researchers hope to define a model of Deqi brain function by applying the brain functional imaging technology, through which the cases fit this model can be defined as Deqi, so it may become easy to distinguish whether to get Deqi or not [39]. Kong et al. [40] have confirmed that the signals of fMRI can correctly reflect the specificity Deqi state between the different individuals, which provides a reliable method to evaluate Deqi.

\section{Conclusion}

Acupuncture is one of the important methods to realize "mediating meridians, regulating the $q i$ and blood" in TCM, in which Deqi plays an important role in the process of acupuncture. Neijing lays the theoretical basis of the theory of Deqi. Deqi does not only refer to needling sensations, but also involves the changes of $q i$ induced by needle insertion into the acupoint. The signs of Deqi include the patient's sensations (needling sensations), objective physiological changes (commonly refer to the skin redness around the acupoint as well as the response of brain), and the acupuncturist's perceptions. Deqi is closely related to the treatment efficacy; however, it is not a necessary sign for the most ideal efficacy. The characteristics of Deqi, including what kind of sensations of both patient and acupuncturist, their prevalence, intensity, time duration, and the propagated sensation along channel will all affect the treatment efficacy. So acupuncturists should pay attention to elicit Deqi and control its state, which is also the key point in modern research on the therapeutic implications of Deqi.

\section{Authors' Contribution}

Hong-Wen Yuan and Liang-Xiao Ma contributed equally to this work.

\section{Acknowledgments}

The authors sincerely acknowledge the National Basic Research Program of China (973 Program) (nos. 2012CB518506 and 2006CB504503), the National Natural Science Foundation of China (no. 30973793), and the Doctoral Program of Higher Education of Ministry of Education of China (no. 20090013110005) for funding this study.

\section{References}

[1] B. Wang, Huang Di Nei Jing, TCM Ancient Books Press, Beijing, China, 2003.

[2] Y. W. Zhu, "Academic strong points and clinical experience of veteran TCM doctor Luo Shirong," Chinese Acupuncture \& Moxibustion, vol. 23, no. 3, pp. 37-39, 2003.

[3] Y. Q. Yuan, "Xie Guorong's experience on needling methods," Chinese Acupuncture \& Moxibustion, vol. 23, no. 11, pp. 44-46, 2003.

[4] X.-Y. Gao, S.-J. Shao, S.-X. Shao, and Q.-L. Ma, "Study on professor Shao Jing-Ming's academic thought," Chinese Acupuncture \& Moxibustion, vol. 27, no. 5, pp. 362-364, 2007.

[5] Q. Wang, R. Hu, X.-Y. Zhong, and G.-Q. Zhu, "Professor HUANG Xuan-Wei's clinical experience in acupuncture and moxibustion," Chinese Acupuncture \& Moxibustion, vol. 28, no. 6, pp. 445-447, 2008.

[6] C. Witt, B. Brinkhaus, S. Jena et al., "Acupuncture in patients with osteoarthritis of the knee: a randomised trial," The Lancet, vol. 366, no. 9480, pp. 136-143, 2005.

[7] W. Takeda and J. Wessel, "Acupuncture for the treatment of pain of osteoarthritic knees," Arthritis Care and Research, vol. 7, no. 3, pp. 118-122, 1994.

[8] J. Vas, E. Perea-Milla, C. Méndez et al., "Efficacy and safety of acupuncture for chronic uncomplicated neck pain: a randomised controlled study," Pain, vol. 126, no. 1-3, pp. 245-255, 2006.

[9] X. Q. Huang, "The study of relationship between acupuncture effect and Deqi phenomenon," Chinese Acupuncture \& Moxibustion, vol. 19, no. 1, pp. 19-21, 1999.

[10] M. H. Sui, C. Y. Wang, and W. Z. Ma, "Effect of arrival of Qi and the method of reinforcing-reducing by twirling and rotating the needle by acupuncturing quchi(LI 11) on the blood pressure 
of essential hypertension patients," Information on Traditional Chinese Medicine, vol. 29, no. 3, pp. 87-90, 2012.

[11] J. Xiong, F. Liu, M.-M. Zhang, W. Wang, and G.-Y. Huang, "De-qi, not psychological factors, determines the therapeutic efficacy of acupuncture treatment for primary dysmenorrhea," Chinese Journal of Integrative Medicine, vol. 18, no. 1, pp. 7-15, 2012.

[12] P. White, P. Prescott, and G. Lewith, "Does needling sensation (de qi) affect treatment outcome in pain? Analysis of data from a larger single-blind, randomised controlled trial," Acupuncture in Medicine, vol. 28, no. 3, pp. 120-125, 2010.

[13] A. Enblom, A. Johnsson, M. Hammar, E. Onelöv, G. Steineck, and S. Börjeson, "Acupuncture compared with placebo acupuncture in radiotherapy-induced nausea-a randomized controlled study," Annals of Oncology, vol. 23, no. 5, pp. 13531361, 2012.

[14] X. B. Chang, X. N. Fan, S. Wang et al., "The origin of 'acupuncture quantification' study," Journal of Traditional Chinese Medicine, vol. 52, no. 5, pp. 363-366, 2011.

[15] A. Benham and M. I. Johnson, "Could acupuncture needle sensation be a predictor of analgesic response?" Acupuncture in Medicine, vol. 27, no. 2, pp. 65-67, 2009.

[16] A. White, M. Cummings, P. Barlas et al., "Defining an adequate dose of acupuncture using a neurophysiological approach-a narrative review of the literature," Acupuncture in Medicine, vol. 26, no. 2, pp. 111-120, 2008.

[17] X. M. Shi, Acupuncture and Moxibustion (Zhen Jiu Xue), China Traditional Medicine Press, Beijing, China, 2007.

[18] X. Cheng, Chinese Acupuncture \& Moxibustion, Foreign Languages Press, Beijing, China, 1987.

[19] Y. X. Yuan, J. Ren, L. Huang et al., Chinese-English Dictionary of Traditional Chinese Medicine, Renmin Weisheng Press, Beijing, China, 1997.

[20] K. Linde, A. Streng, S. Jürgens et al., "Acupuncture for patients with migraine: a randomized controlled trial," Journal of the American Medical Association, vol. 293, no. 17, pp. 2118-2125, 2005.

[21] D. Melchart, A. Streng, A. Hoppe et al., "Acupuncture in patients with tension-type headache: randomised controlled trial," British Medical Journal, vol. 331, no. 7513, pp. 376-379, 2005.

[22] K. K. S. Hui, E. E. Nixon, M. G. Vangel et al., "Characterization of the "Deqi" response in acupuncture," BMC Complementary and Alternative Medicine, vol. 7, article 33, 2007.

[23] X. J. Xia, J. G. Xie, and S. Q. Zhang, "A test on composing time of Huang Di Nei Jing," Gansu Journal of Traditional Chinese Medicine, vol. 22, no. 5, pp. 4-5, 2009.

[24] G. Z. Chen, Huai Nan Zi, Zhonghua Book Company, Beijing, China, 2012.

[25] M. Feng and T. S. Hou, "A rough vision for Qizhi in Huang Di Nei Jing," Jiangsu Journal of Traditional Chinese Medicine, vol. 42 , no. 12 , pp. 66-67, 2010.

[26] C. Z. Liu and S. Z. Gao, "Discussion on arrival of Qi in the miraculous pivot," Liaoning Journal of Traditional Chinese Medicine, vol. 30, no. 3, pp. 173-174, 2003.

[27] Nanjing College of Traditional Chinese Medicine, Collation and Annotation of Nanjing, People's Medical Publishing Press, Beijing, China, 2009.

[28] J. Y. Li, "A test on composing time of Nanjing," Journal of Henan University of Traditional Chinese Medicine, no. 4, pp. 12-14, 1979.
[29] J. Z. Yang, Zhen Jiu Da Cheng, People's Medical Publishing Press, Beijing, China, 2006.

[30] C. Y. Wang, M. Xue, Q. G. Liu et al., "The difference understanding about "Deqi" and "Qizhi” between Neijing and Nanjing," Journal of Beijing University of Traditional Chinese Medicine, vol. 14, no. 3, pp. 28-29, 2007.

[31] Z. G. Li and S. K. Liu, "Discussion on the "arrival of Qi" in medical classic 'the yellow emperor's internal classic," Acupuncture Research, vol. 30, no. 4, pp. 246-248, 2005.

[32] P.-Q. Wang, "Deqi and application and manipulation of Deqi," Chinese Acupuncture \& Moxibustion, vol. 27, no. 5, pp. 349-351, 2007.

[33] F. Zhang and H. D. Wang, "Discussion on needling sensation, arrival of qi and needling response (Deqi)," Chinese Acupuncture \& Moxibustion, vol. 32, no. 12, pp. 1132-1134, 2012.

[34] C. A. Vincent, P. H. Richardson, J. J. Black, and C. E. Pither, "The significance of needle placement site in acupuncture," Journal of Psychosomatic Research, vol. 33, no. 4, pp. 489-496, 1989.

[35] H. Park, J. Park, H. Lee, and H. Lee, "Does Deqi (needle sensation) exist?" American Journal of Chinese Medicine, vol. 30, no. 1, pp. 45-50, 2002.

[36] J. Kong, R. Gollub, T. Huang et al., "Acupuncture Deqi, from qualitative history to quantitative measurement," Journal of Alternative and Complementary Medicine, vol. 13, no. 10, pp. 1059-1070, 2007.

[37] P. White, F. Bishop, H. Hardy et al., "Southampton needle sensation questionnaire: development and validation of a measure to gauge acupuncture needle sensation," Journal of Alternative and Complementary Medicine, vol. 14, no. 4, pp. 373-379, 2008.

[38] J. J. Park, M. Akazawa, J. Ahn et al., "Acupuncture sensation during ultrasound guided acupuncture needling," Acupuncture in Medicine, vol. 29, no. 4, pp. 257-265, 2011.

[39] X.-S. Lai and Y. Huang, "A cerebral functional definition on the specificity of acupoints, needling sensation and association of acupoints based on the 'acupoints-brain relation hypothesis"' Chinese Acupuncture \& Moxibustion, vol. 27, no. 10, pp. 777-780, 2007.

[40] J. Kong, R. L. Gollub, J. M. Webb, J.-T. Kong, M. G. Vangel, and K. Kwong, "Test-retest study of fMRI signal change evoked by electroacupuncture stimulation," NeuroImage, vol. 34, no. 3, pp. 1171-1181, 2007. 


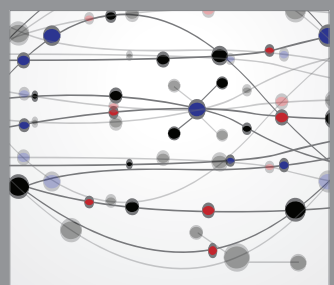

The Scientific World Journal
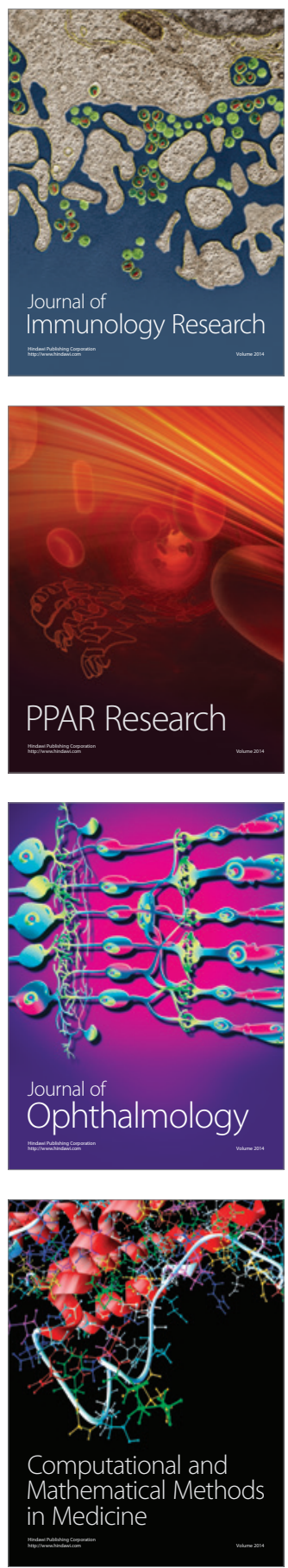

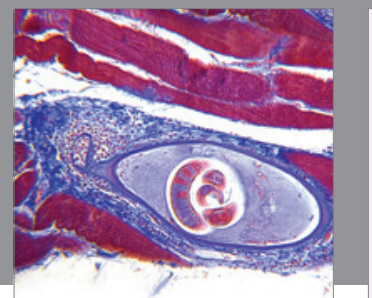

Gastroenterology

Research and Practice
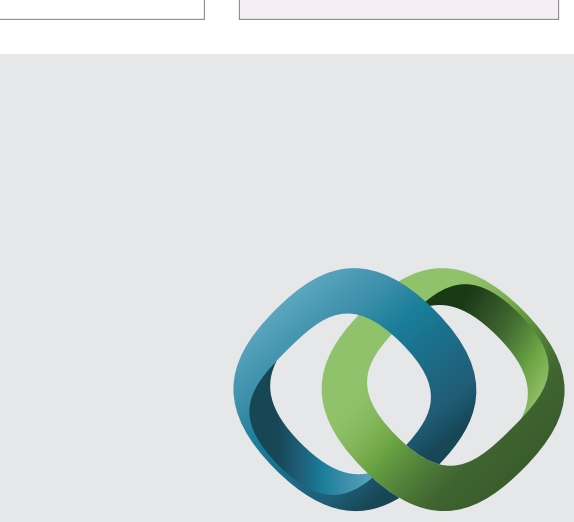

\section{Hindawi}

Submit your manuscripts at

http://www.hindawi.com
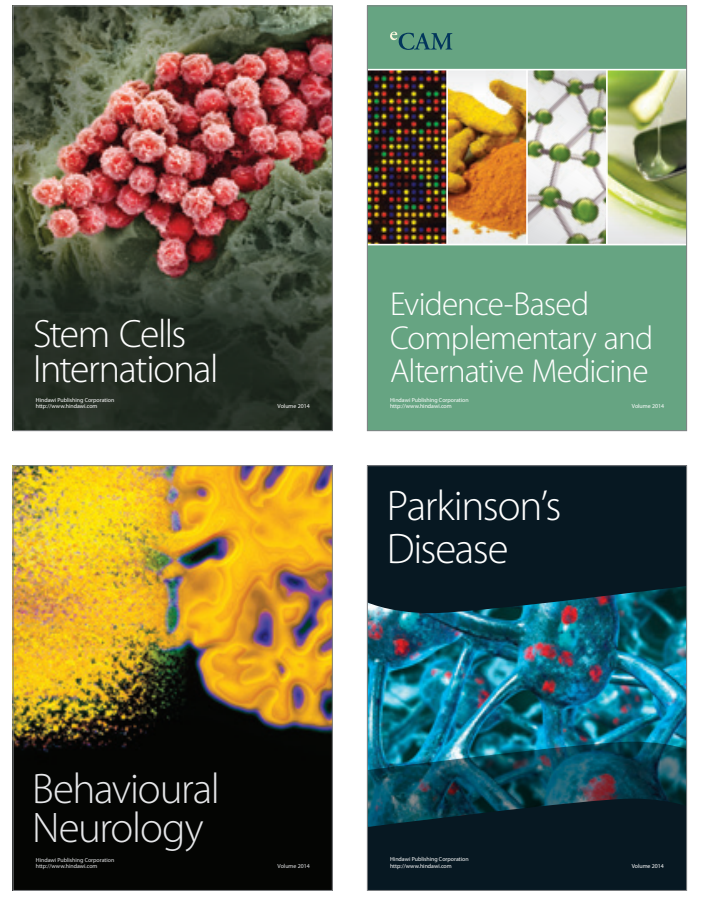
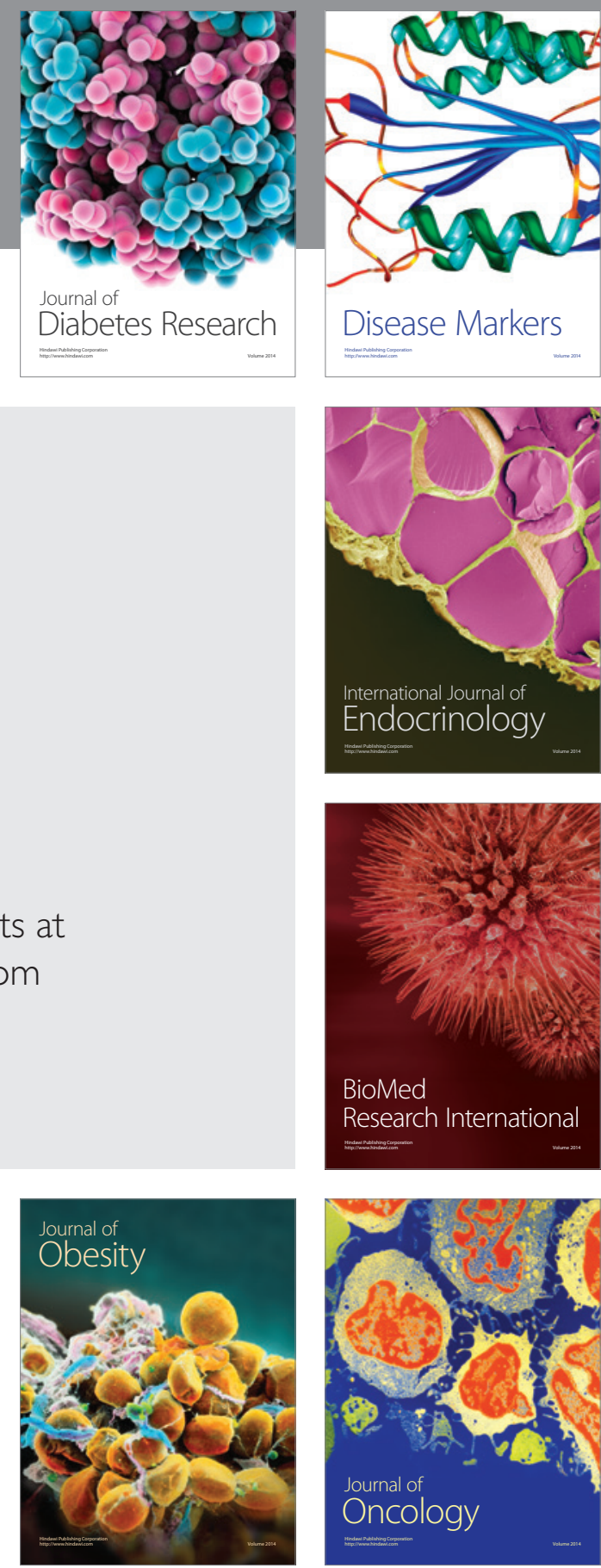

Disease Markers
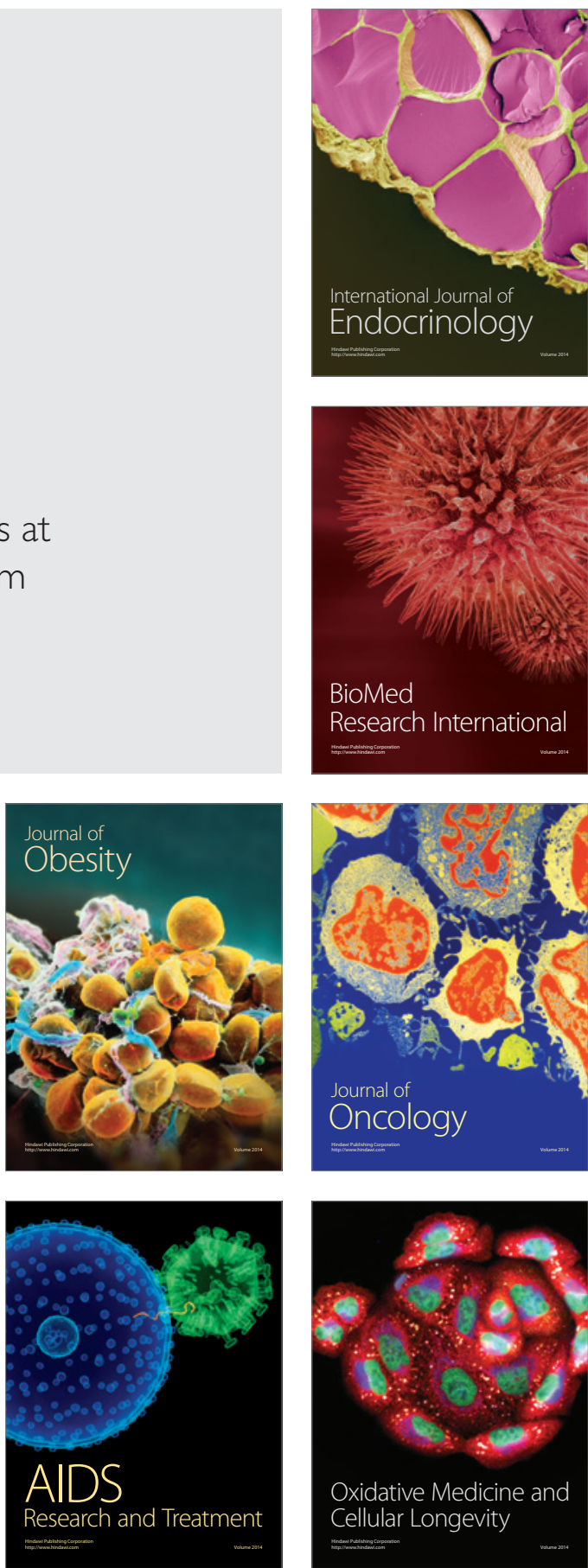